\title{
Two blocks connected by a string with variable tension: A dynamic case.
}

\author{
H. J. Herrera-Suárez ${ }^{1, a}$ \& M. Machado-Higuera ${ }^{1, b}$ \& J. H. \\ Muñoz ${ }^{2, c}$ \\ ${ }^{1}$ Universidad de Ibagué, Facultad de Ciencias Naturales y Matemáticas, \\ Ibagué-Colombia, Carrera 22 Calle 67, barrio Ambalá \\ ${ }^{2}$ Universidad del Tolima, Departamento de Física, Ibagué-Colombia, Barrio Santa \\ Helena Parte Alta. \\ E-mail: ${ }^{a}$ hernan.herrera@unibague.edu.co \& ${ }^{b}$ \\ maximiliano.machado@unibague.edu.co \& ${ }^{c}$ jhmunoz@ut.edu.co
}

\begin{abstract}
In this paper, the dynamic case of a system made up of two blocks connected by a string over a smooth pulley is revisited. One mass lies on a horizontal surface without friction, and the other mass has a vertical displacement. The motion equation is obtained and its solution is determined using the Mathematica package. Also an experimental montage for this system is made and experimental data for the vertical position $y$ in function of the time $t$ are obtained using a Data Acquisition System and the Tracker video analysis. The relation $y$ vs $t$ can be represented by a polynomial of degree six. An average relative error of 3.61(10.14)\% is obtained between the theoretical results acquired with Mathematica and the data taken from the Tracker (Data Acquisition System).
\end{abstract}

Keywords: newtonian mechanics; tracker; data acquisition system.

\section{Introduction}

In this work, a system of two blocks of masses $m_{1}$ and $m_{2}$ connected by a string over a smooth pulley and subjected to variable acceleration is studied. The mass $m_{2}$ is suspended, and the mass $m_{1}$ moves on a horizontal surface without a coefficient of dynamic friction. The string is extensionless, and uniform, and its mass is negligible. Figure 1 shows the forces acting on this system.

Unlike other systems made up of two masses tied to a rope that passes through a pulley (such as those shown in Figure 2), this problem is significant because the tension $T$, the normal force $N$ and the acceleration are not constant because they depend on the variation of the angle $\theta$. This system is a more complex progression of the four common configurations shown in Figure 2.

The static situation of this problem has been studied previously in some fundamental physics textbooks [1-6], in several papers [7-12] and on the website of A. Franco [13. The dynamic case, in which there is no friction between the horizontal 


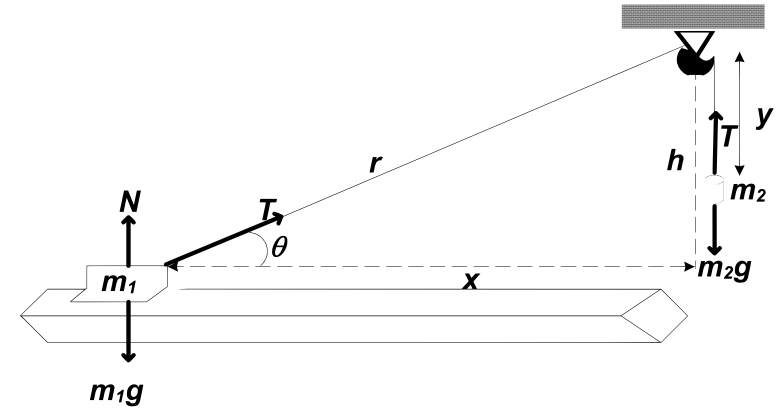

Figure 1: Two blocks tied to an extensionless string.
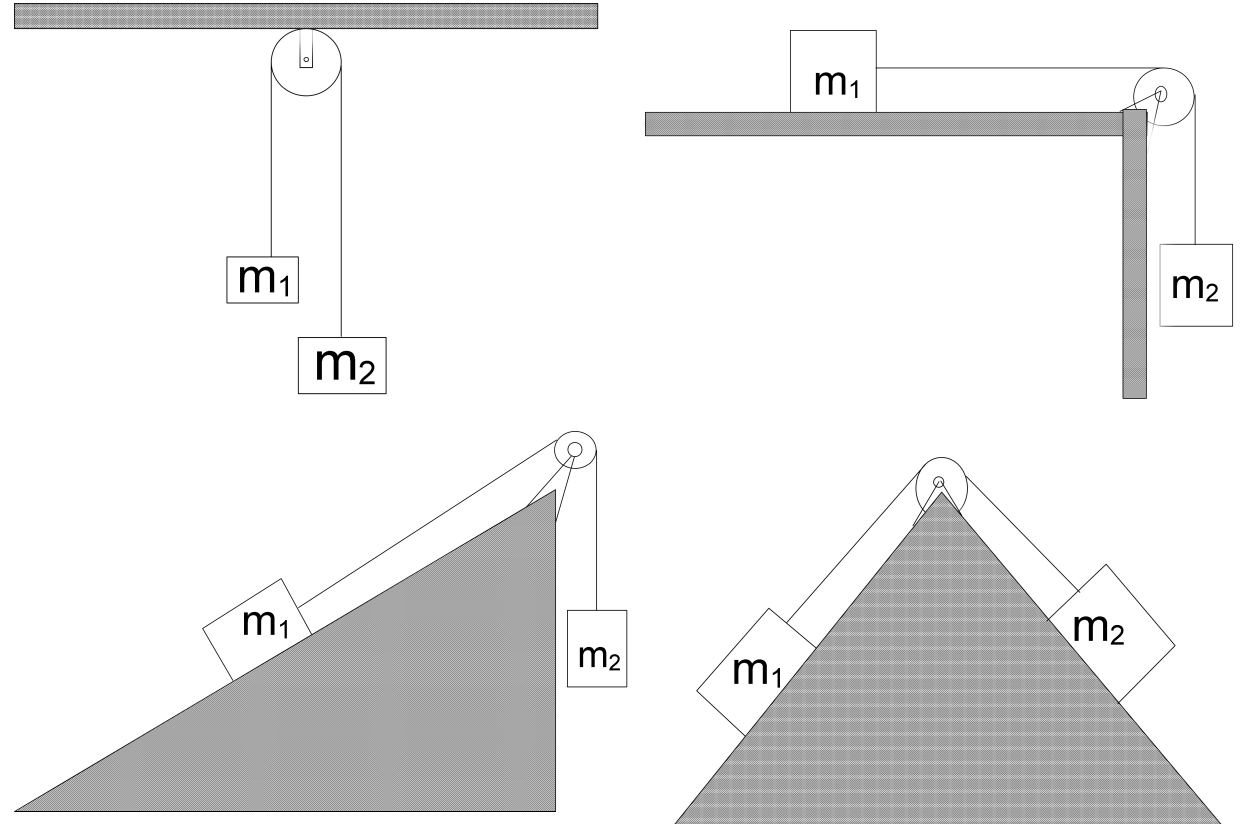

Figure 2: Systems made up of two masses tied to a rope that passes through a pulley. The tension and the acceleration are constant.

surface and the mass $m_{1}$, was proposed as a problem in Serway-Jewett's physics textbook [1] and its solution appears in the Instructor's Solution Manual of the same author [2]. In this paper, this case is revisited with the purpose of performing a complete analysis to this system. The motion equation is explicitly obtained, and its solution is found using the Mathematica package. An experimental montage is made, and experimental data for the position in function of the time for the mass $m_{2}$ are got using a Data Acquisition System (DAS) and the Tracker video analysis. The experimental results are then compared with the theoretical solution.

This paper is organized as follows. In Section 2, the experimental arrangement built by the authors to represent the system shown in Figure 1 is displayed; in Section 3 , the theoretical analysis is presented, and the results are briefly discussed in Section 4. Finally, in Section 5, some concluding remarks are given. 


\section{Experimental arrangement}

Figure 3 shows the experimental arrangement used to represent the system in Figure 1. The two masses are $m_{1}=574.12 \mathrm{~g}$ and $m_{2}=100 \mathrm{~g}$ (measured with a WTC 2000 precision balance [20]), and the length of the string is $l=r+y=2.09 \mathrm{~m}$ and $h=0.98 \mathrm{~m}$. The mass $m_{2}$ moves down in the vertical direction, and the mass $m_{1}$ moves horizontally over the linear air track, reference 11202 - 88, of the company Phywe [14]. The DAS used is made up of: a sensor-CASSY 2, reference 524013 [15]; a timer S [16]; a multi core cable, 6 pole, $1.5 \mathrm{~m}$, reference 50116 [17]; a combination spoked wheel, reference 337464 [18]; and a combination light barrier, reference 337462 [19], of the company Phywe, and a computer, which are fundamental to the acquisition of data.

The air supply (see (i) in Figure 3) in used to reduce the friction between the mass $m_{1}$ and the air track. The data for the variable $y$ in function of the time were registered for the DAS with the initial condition $y(0)=-0.255 \mathrm{~m}$. The movement of the two masses was also recorded with a smartphone, and the video was analized using the Tracker video analysis [21].

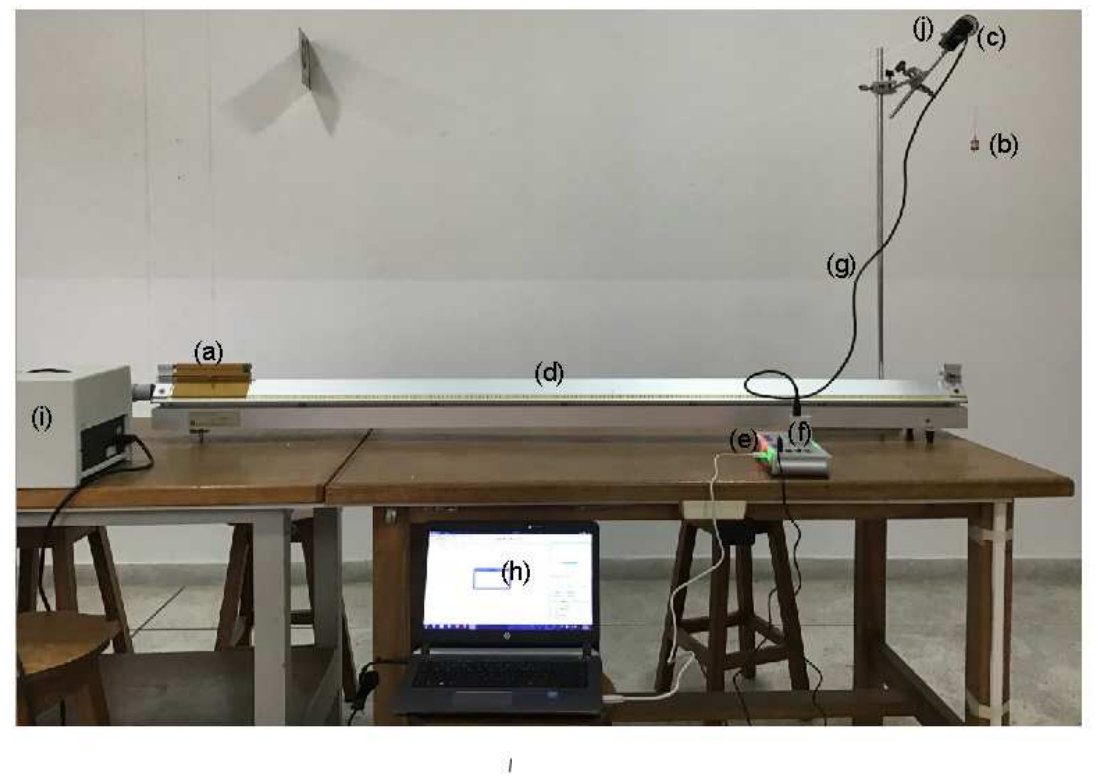

Figure 3: Experimental arrangement: (a) mass $m_{1}$; (b) mass $m_{2}$; (c) combination spoked wheel; (d) air track; (e) sensor CASSY 2; (f) timer S; (g) multi core cable, 6 pole, $1.5 \mathrm{~m}$; (h) computer; (i) air supply, and (j) combination light barrier.

\section{Theoretical analysis}

The system displayed in Figure 1 is analyzed using Newton's second law. The sum of the forces for the particle $m_{1}$ in the $x$ direction is 
Two blocks connected by a string with variable tension: A dynamic case.

$$
T \cos \theta=m_{1} a_{x} ;
$$

and the sum of the forces for the mass $m_{2}$ in the $y$ direction gives

$$
T-m_{2} g=-m_{2} a_{y} .
$$

From this equation, the tension $T$ is obtained and by substituting it in Equation (11) the following is got

$$
\left(m_{2} g-m_{2} a_{y}\right) \frac{x}{\sqrt{x^{2}+h^{2}}}=m_{1} a_{x},
$$

where $\cos \theta=x / r=x / \sqrt{x^{2}+h^{2}}$ (according to Figure 1).

Considering Figure 1, the length $l$ of the rope is given by

$$
l=\sqrt{x^{2}+h^{2}}+y .
$$

From this equation the following is obtained

$$
x=\sqrt{(l-y)^{2}-h^{2}} .
$$

On the other hand, if differentiated with respect to time in Equation (44), the equation below is got

$$
v_{y}=-x v_{x}\left(x^{2}+h^{2}\right)^{-\frac{1}{2}},
$$

where $v_{x}=\frac{d x}{d t}$ and $v_{y}=\frac{d y}{d t}$ are the speeds of mass $m_{1}$ and $m_{2}$, respectively.

Now, by differentiating this expression with respect to time, after replacing $x$ and $v_{x}$, by means of the Equations (5) and (6), respectively, the below expression is got

$$
a_{x}=-\left(\frac{h^{2} v_{y}^{2}}{\left((l-y)^{2}-h^{2}\right)^{\frac{3}{2}}}+\frac{(l-y) a_{y}}{\left((l-y)^{2}-h^{2}\right)^{\frac{1}{2}}}\right),
$$

where $a_{x}=\frac{d v_{x}}{d t}$ and $a_{y}=\frac{d v_{y}}{d t}$ are the accelerations of mass $m_{1}$ and $m_{2}$, respectively. If $v_{y}=0$, then $a_{x}=-u a_{y}$ with $u=(l-y)\left((l-y)^{2}-h^{2}\right)^{-\frac{1}{2}}$. This result agrees with the one presented in the references [1,2].

By Replacing the last expression for $a_{x}$ in Equation (3), the following expression is obtained:

$$
a_{y}=\frac{m_{2} g\left((l-y)^{2}-h^{2}\right)^{2}+(l-y) m_{1} h^{2} v_{y}^{2}}{\left((l-y)^{2}-h^{2}\right)\left(m_{2}\left((l-y)^{2}-h^{2}\right)-m_{1}(l-y)^{2}\right)} .
$$

This differential equation gives the equation motion for $y$. 


\section{Results and discussion}

The evolution of the variable $y$ in function of the time $t$ has been obtained via three different ways: (i) employing the Tracker, (ii) manipulating the DAS, and (iii) using the Mathematica package.

As mentioned in Section 2, the movement of the masses $m_{1}$ and $m_{2}$ were recorded with a smartphone and the video was analyzed with the Tracker. Figure 4 (see left side) shows a picture of the movement of the mass $m_{2}$ using this computational tool. The vertical displacement of the particle $m_{2}$ in function of the time, $y$ vs $t$, is displayed in the top-right of this figure, and the data are shown in the bottom-right. Figure 5 shows the graph of $y$ vs $t$ obtained with the Tracker (see the solid black circles). On the other hand, the software CASSY was also used to obtain $y$ vs $t$ from the DAS (see the solid blue circles).
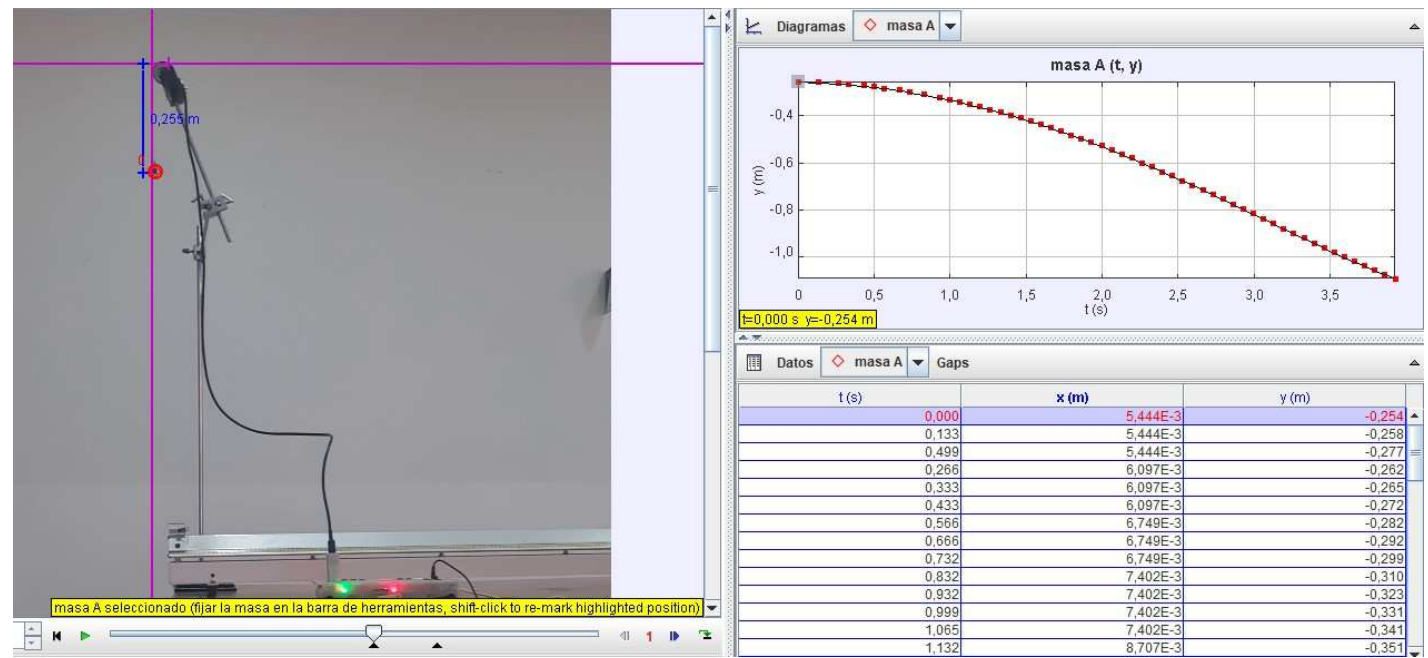

Figure 4: An image of the movement of the mass $m_{2}$ using the Tracker.

The solution of the differential equation shown in Equation (8), was obtained by means of the Mathematica, using the commands NDSolve and Plot[Evaluate[y[t] /. s, t, 0, 1]. This solution is displayed in Figure 5 (see the solid red circles).

Figure 5 was obtained using the OriginPro package [22]. This software allows the results obtained by the Tracker, the DAS, and Mathematica to be presented in a single image. The experimental data confirm that the motion of the mass $m_{2}$ is neither uniform nor uniformly accelerated. 


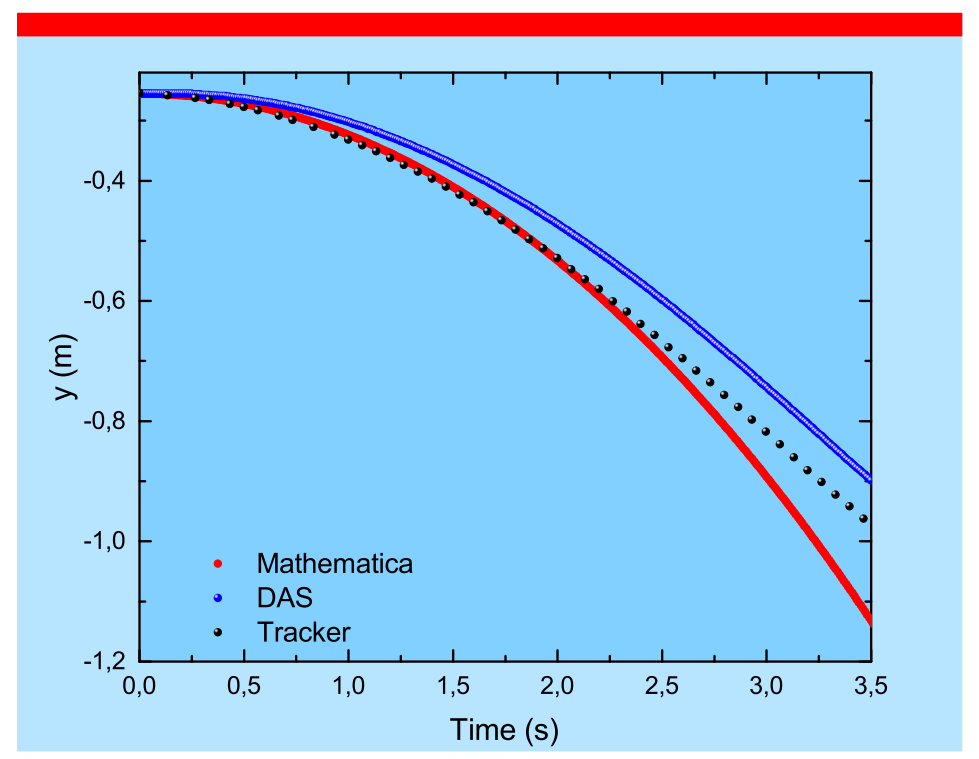

Figure 5: Graph of $y$ vs $t$ for the mass $m_{2}$. The solid black (blue) circles represent the experimental data obtained with the Tracker (DAS). The red circles represent the solution of the differential equation obtained with Mathematica.

The obtained data with the DAS and the Tracker and the acquired result with Mathematica, were fitted by means of the OriginPro. The best fit is given by a polynomial of degree six with a correlation coefficient of, approximately, 0.999. The corresponding expressions are display in the Table 1 . The curves and the errors shown in Figure 6 were obtained from these equations. The results obtained with Mathematica and the Tracker (DAS) were compared (see Figure 6a (Figure 6b)), giving that the average relative error of the theoretical results acquired with Mathematica in relation to the data taken with the Tracker and the DAS is $3.61 \%$ and $10.14 \%$, respectively.

It is important to note that both measured curves indicate lower accelerations than the calculated indicating that there is some small friction. Further, the acceleration obtained with the DAS is slower than the one obtained with the Tracker probably because of cord slippage around the pulley. The curves show good agreement at the beginning and then diverge because the increasing angle is altering whatever friction there is. 


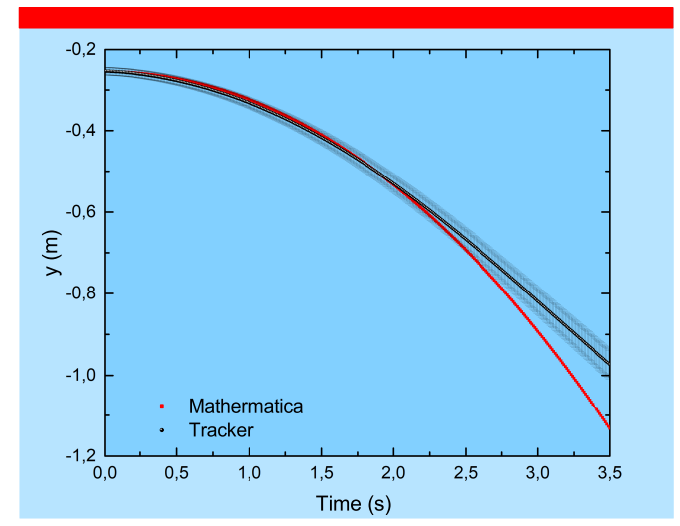

(a)

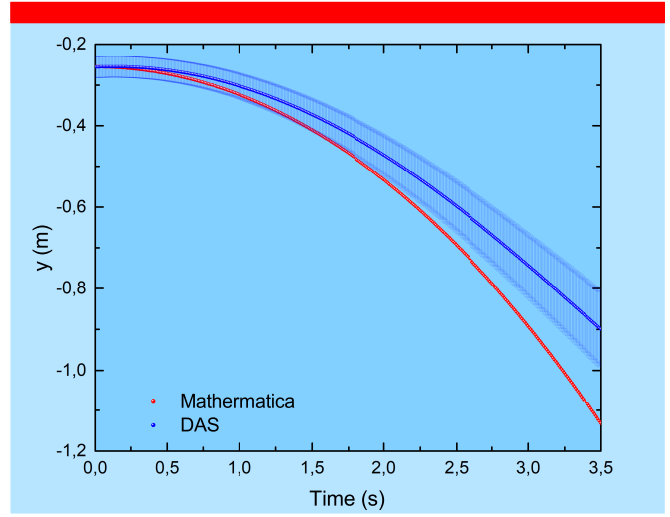

(b)

Figure 6: Comparison of the obtained results from Mathematica and the Tracker (a) and the DAS (b).

Table 1: $y$ vs $t$ for the obtained data with the Tracker, the DAS and the theoretical solution given by Mathematica.

\begin{tabular}{lcl}
\hline & \\
& $y=\quad$ & $-1.45919 \times 10^{-6} t^{6}+5.66164 \times 10^{-5} t^{5}-$ \\
& $1.76691 \times 10^{-4} t^{3}-0.06829 t^{2}$ \\
& $+2.75608 \times 10^{-5} t-0.255$ \\
Mathematica $\quad y=$ & $1.60902 \times 10^{-4} t^{6}-0.00132 t^{5}+0.00502 t^{4}$ \\
& & $-0.00811 t^{3}-0.0576 t^{2}+0.01529 t-0.25567$ \\
\hline \multirow{2}{*}{ DAS } & $y=$ & $-1.75581 \times 10^{-4} t^{6}+0.00213 t^{5}-0.00773 t^{4}$ \\
& $+0.01381 t^{3}-0.07397 t^{2}-0.0118 t-0.25406$ \\
\hline \multirow{2}{*}{ Tracker } &
\end{tabular}

\section{Concluding remarks}

The authors studied the dynamic situation of a block of mass $m_{1}$ on a horizontal plane being pulled at an angle $\theta$ with the horizontal by a tension due to a suspended mass $m_{2}$, without considering the friction between $m_{1}$ and the horizontal plane. The experimental results were obtained using the Tracker and the DAS shown in Figure 3, and the theoretical solution to the motion equation was found using Mathematica.

The movement of the mass $m_{2}$ along the y-axis is neither uniform nor uniformly accelerated. The best fit for $y$ in function of the time is a polynomial of degree six. The theoretical prediction obtained with Mathematica gives a better agreement with data taken using the Tracker than using the DAS.

This work is worthwhile because it allows the articulation between experiment and theory, facilitating students' understanding of the physics behind the theory. Besides, it allows one to check the experimental viability of theoretical problems proposed in physics textbooks. 
Two blocks connected by a string with variable tension: A dynamic case.

\section{References}

[1] Serway R A and Jewett J W 2014 Physics for Scientists and Engineers 9th ed vol 1 Boston: Cengage Learning)

[2] Serway R A and Jewett J W 2014 Physics for Scientists and Engineers with Modern Physics Instructor Solutions Manual 9th ed (Boston: Cengage Learning)

[3] González F A and Martínez Hernández M 1978 Problems in general Physics 1st ed (Tebar Florez In Spanish)

[4] Ohanian H C and Markert J T 2009 Physics for Engineers and Scientists 3rd ed (W W Norton Company)

[5] Hibbeler R C 2010 Engineering Mechanics - Dynamics 12th ed (Prentice Hall)

[6] Riley W F and Sturges L D 1996 Mechanical engineering: dynamic 1st ed (Reverté In Spanish)

[7] Lerman N 1964 Open-Ended Problem Instruction in General Physics Am. J. Phys. 32 (12) 927-936

[8] Mak S 1987 Extreme values problems in mechanics without calculus Am. J. Phys. 55 (10) 929-931

[9] Sütt D 1994 Elementary discussion of an optimization problem concerning friction Phys. Educ. 29 (4) 249-252

[10] van den Berg W H 2000 The best angle for dragging a box Phys. Teach. 38 (8) 506-508

[11] Leonard W J 2001 Dragging a Box: The representation of constraints and the constraint of representations Phys. Teach. 39 (7) 412-414

[12] Benitez D, Herrera-Suárez H J and Muñoz J H 2019 Symmetry in the system conformed by two blocks connected by a string with variable tension Rev. Cub. Phys. 36 (2) 139-143

[13] García F A [Online] The best angle to drag a block http://www.sc.ehu.es/sbweb/fisica3/dinamica/arrastra/arrastra.html

[14] http://www.phywe.com/en/air-track-complete.html

[15] https://www.leybold-shop.com/physics/physics-equipment-old/heat/temperaturemeasurement/digital-thermometers/with-din-plug/sensor-cassy-2-524013.html

[16] https://www.leybold-shop.com/physics/physics-equipment/systems/cassy/sensor-boxessensors/physics/timer-s-524074.html

[17] https://www.leybold-shop.com/physics/physics-equipment-old/electricity-electronics/electricityand-electronics-accessories/connecting-leads-and-small-accessories/multi-core-cable-6-pole-1-5m-50116.html

[18] https://www.leybold-shop.com/combination-spoked-wheel-337464.html

[19] https://www.leybold-shop.com/physics/physics-equipment-old/mechanics/dynamics-andkinematics/recording-of-motions/with-light-barrier/combination-light-barrier-337462.html

[20] https://radwagusa.com/en/wtc-2000-precision-balance,w1,5W1,101-102-109\#1

[21] https://physlets.org/tracker/ Tracker: Copyright (c) 2020 Douglas Brown

[22] https://www.originlab.com 